\title{
A multi-scenario Decision Support System for real-time operation of over-year multi-reservoir systems 1. Model structure
}

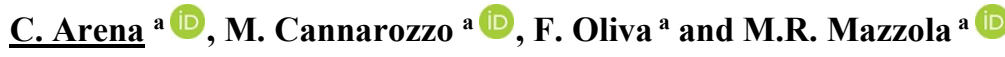 \\ ${ }^{a}$ Dipartimento di Ingegneria, Università degli Studi di Palermo, Italy \\ Email: claudio.arena@unipa.it
}

\begin{abstract}
Water resources systems for water supply with over-year behaviour are widespread in many areas of the world, including Southern Europe, Middle East, China, Western US, South Africa and Australia. Although in these systems the risk of highly variable inflows is partially internalized in the design process by letting these reservoirs have large storage capacities, operation of these systems is challenged by changing demand levels, changing purposes and objectives, and climate change. Given their dimensions, these systems are often managed in a centralized fashion, with one authority planning allocations to different users. As inadequate management may operate the system either too conservatively or in a fashion unaware of the occurrence of long, multi-year dry spells, the decision-making process should be supported by appropriate decision support tools. When used on a regular basis, in a real-time mode, by updating current information on water resources status and using inflow forecasts, such support tools should be able to identify risky situations suitably in advance, suggest appropriate hedging policies and optimize the use of additional, costly, water resources that can help mitigate the impacts of sustained dry periods.
\end{abstract}

One of the key factors for a successful management is obviously the ability to predict future inflows into the system suitably in advance. Presently, the state of the art of climate services is provided by seasonal climate forecasts over a six-month horizon, coupled with downscaling models to turn climate input into streamflow. Forecasts for further-reaching time periods are an active field of research, but are not currently used by industry. However, also downscaled seasonal forecasts are out of reach for most water agencies and utilities, as they imply the availability of ad-hoc skills, resources and facilities to acquire climate forecasts and turn them into inflow forecasts. It hence makes sense to develop simpler, data-driven forecast systems to be integrated into the decision support tools to improve the quality of decisions, while research progresses, and resources and skills for implementing hydrological forecast are being developed

To this end, in this paper we introduce a multi-scenario mathematical programming (algebraic) model for real time management of a multi-reservoir system for water supply where consideration of a multi-year Forecasting Horizon (FH) is necessary given the physical features of the system. It is a linearized MIP (mixed integer programming) model that optimizes water allocations to municipal and irrigation demand centres driven by the objective of minimizing weighted total discounted costs (scarcity costs plus costs of additional water resources) along the multi-year FH, being the weights the occurrence probability of each of the three inflow scenarios. Constraints include 1) mass balances at system's nodes, 2) systems' topology, 3) component's capacity, 4) spills, 5) non-empty conditions on reservoir storage at the end of the FH. It is a multi-scenario optimization tool because future, uncertain inflows are modelled, until the end of the current water year, as three different inflow scenarios: low flows, normal flows and high flows. The optimization model is solved for the three different scenarios and a unique solution that can be turned into one actual, implementable decision at the present time step is obtained by imposing non-anticipatory (or congruity) constraints according to the principle of scenario aggregation. Given the over-year nature of the systems of interest for this study, the time unit is one month.

This paper is devoted to the description of the structure of the DSS, of the procedures followed to estimate scarcity costs and of the techniques used to linearize this partly non-linear model. Construction and estimation of scenarios as well as results of DSS simulation to evaluate its performances are the object of a companion paper.

Keywords: Decision Support Systems, real time reservoir system management, scenario optimization 
Arena et al., A multi-scenario Decision Support System for real-time operation of over-year multi-reservoir systems 1. Model description

\section{INTRODUCTION}

Water resources systems for water supply with over-year behaviour are widespread in many areas of the world, including Southern Europe, Middle East, China, Western US, South Africa and Australia. Although in these systems the risk of highly variable inflows is partially internalized in the design process by letting these reservoirs have large storage capacities, operation of these systems is challenged by changing demand levels, changing purposes and objectives, and climate change. Given their dimensions, these systems are often managed in a centralized fashion, with one authority planning uses and allocations to different users. As inadequate management may operate the system either too conservatively or in a fashion unaware of the occurrence of long, multi-year dry spells, the decision making process should be supported by appropriate decision support tools. When used on a regular basis, in a real-time mode, by updating current information on water resources status and using inflow forecasts, such support tools should be able to identify risky situations suitably in advance, suggest appropriate hedging policies and optimize the use of additional, costly, water resources that can help mitigate the impacts of sustained dry periods. One of the key factors for a successful management is the ability to predict future, uncertain, inflows into the system suitably in advance. Presently, the state of the art of climate services are seasonal climate forecasts over a six-month horizon, coupled with downscaling models to turn climate input into streamflow (Yuan et al. 2015). Forecasts for further-reaching time periods are an active field of research (Smith et al. 2016, Dariane et al. 2018), but are not currently used by industry. However, also downscaled seasonal forecasts are out of reach for most water agencies and utilities: it hence makes sense developing data-driven forecast systems to be integrated into the DSS to improve the quality of decisions, while research progresses, and resources and skills for implementing hydrological forecast are being developed. In this paper we introduce a multi-scenario MIP (Mixed Integer Programming) model for real time management of a multi-reservoir system where future inflows until the end of the current water year are modelled as scenarios conditioned by the past history of inflow from the beginning of the water year to the current month. The resulting stochastic optimization problem is solved using the scenario aggregation principle (Wets 1989). The model presented here is the prototype and test-bench of a larger model to support of operations of the water resources system providing water supply to over 4,000,000 persons and over 50,000 hectares of irrigated agriculture in Apulia, in southern Italy. Literature review has shown that although a number of different scenario-based models have been developed, most of them focus on hydropower production (e.g. Xu et al. 2015) or shorter forecasting horizons than those need for this type of systems. In addition, explicit incorporation of non-linear scarcity costs in the decision-making framework, as introduced in this model, is also uncommon, while it may prove useful to provide an objective, straightforward criterion for water resources allocation among different use types. Further information on scenario construction and scenario inflows estimation, as well as the description of DSS application on a real-world, two reservoir system in Southern Italy is provided in a companion paper (Arena et al. 2019).

\section{THE MODEL}

In a real-time, dynamic management context, the optimization model aims at minimizing total costs (scarcity costs plus the costs of additional water resources) over a multi-year Forecasting Horizon (FH) to produce a decision on water allocations and intakes of additional water for the Decision Horizon (DH), identified with the current month. In doing this, the model must account for system's constraints and makes use of some type of inflow forecast. Instead of using exogenous forecasts, in the proposed model inflows are provided under the form of scenarios: the outcomes of the different scenarios are then turned into a unique decision using the Scenario Aggregation principle (Wets 1989). Both scenario construction and the methodologies for the estimation of scenario probabilities and inflows are described in the companion paper (Arena et al. 2019). Here it suffices to anticipate that three inflow scenarios are considered: a low, a normal and a high one.

\subsection{The objective function}

In the objective function, each of the scenarios is weighted by its probability $\operatorname{Prob}_{(s c)}$ :

$C_{t o t}=\sum_{s c=1}^{3} \operatorname{Prob}_{(s c)} \cdot\left[\sum_{i=1}^{N m i n} \sum_{t=1}^{F H} \frac{1}{\left(1+r_{m}\right)^{t}} \cdot S C_{m u n(i, t, s c)}+\sum_{i=1}^{N i r r} \sum_{y r=1}^{F H} \frac{1}{\left(1+r_{y r}\right)^{y r}} \cdot S C_{i r r(i, y r, s c)}+\sum_{i=1}^{N a d d} \sum_{t=1}^{F H} \frac{1}{\left(1+r_{m}\right)^{t}} \cdot C_{a d d(i, t, s c)}\right]$

In (1), $C_{\text {tot }}$ is the total cost, $S C_{\text {mun(i,t,sc }}$ is the scarcity cost borne by the i-th municipal demand centre in inflow scenario sc at month $\mathrm{t}$ of the $\mathrm{FH}, S C_{i r r(i, y r, s c)}$ is the scarcity cost borne by the i-th irrigation water demand centre in scenario sc, in the y-th year of the multi-year FH. $C_{a d d(i, t, s c)}$ is the cost of the additional water resource taken 
Arena et al., A multi-scenario Decision Support System for real-time operation of over-year multi-reservoir systems 1. Model description

from the i-th source of additional water, in scenario sc, at month $\mathrm{t}$ of the $\mathrm{FH} . \mathrm{r}_{\mathrm{m}}$ and $\mathrm{r}_{\mathrm{y}}$ are respectively the monthly and yearly factors that are necessary to discount future costs over the FH to present value. It should be observed that, unlikely costs borne by municipal users, costs borne by agriculture are expressed in annual terms. This is consistent with the fact that water deficits to irrigation will ultimately impact production of crops that take several months to grow and yield harvest, so that it would make little sense basing evaluation on a monthly scale.

The methodologies to estimate scarcity costs are illustrated in section 2.2 , here it is important to stress that according to the principle of scenario aggregation water supplied to the different uses in the Decision Horizon DH (the current month) must be the same regardless of the inflow scenario considered. This is obtained through the non-anticipatory constraint:

$$
\text { Supply }_{(i, s c 1, D H)}=\text { Supply }_{(i, s c 2, D H)}=\text { Supply }_{(i, s c 3, D H)}
$$

\subsection{Assessing scarcity costs}

Scarcity costs are the costs borne by users for not being able to use as much water as demanded. Scarcity costs are hence related to the value of water resources and may be assessed from the demand curve for (a certain use of) water, that relates water demand (consumption) to marginal benefits (unit price, unit productivity, depending on the sector considered). As far as municipal uses are concerned, hundreds of empirical studies carried out from the ' 60 to date have shown that, regardless of the methodologies used to estimate the demand curve, water demand can be considered inelastic to price (i.e. with elasticity $\eta<1$ ). Starting from the above evidence, point expansion methodologies (Griffin 2007) are a straightforward way to assess the price-demand relationship for municipal water using one known point of the curve and assuming a specification for the pricedemand relationship, and an elasticity $\eta$. The known point corresponds to a situation in which there is no water shortage and water supply equals target $T D_{\text {mun }}$. In this case, the water tariff $\mathrm{P}_{\mathrm{T}}$ coincides with the opportunity cost of all the goods needed to produce drinking (municipal) water and can be considered coincident with marginal benefits. The specification is a Cobb-Douglas function $\mathrm{P}=a \cdot \mathrm{Q}^{b}$ that assumes constant elasticity. Q is the aggregated water consumption (in $\mathrm{m}^{3} / \mathrm{month}$ ) by final users at the municipality scale or of a group of municipalities, $\mathrm{P}\left(€ / \mathrm{m}^{3}\right)$ is the "price" of water (the marginal benefit from consuming quantity Q) and $b=1 / \eta$. Parameter $a$ may be estimated from the evidence that when $\mathrm{Q}=T D_{\text {mun }} \cdot(1-L)$, then $\mathrm{P}=\mathrm{P}_{\mathrm{T}} . L$ are water losses in distribution networks that must be considered in order to turn water withdrawn from the supply sources to water consumed by final users.

A consumption less than target $T D_{\text {mun }} \cdot(1-L)$ produces a water deficit and ultimately results in a loss of benefit due to water scarcity that is called scarcity cost. By integration and simple passages, one finds (Arena et al. 2014)

$$
S C_{\operatorname{mun}(i, t, s c)}=a \cdot \frac{\eta}{1+\eta} \cdot\left\{\left[T D_{\operatorname{mun}(i, t)} \cdot(1-L)\right]^{\frac{1+\eta}{\eta}}-\left[\left(T D_{\operatorname{mun}(i, t)}-D e f_{\operatorname{mun}(i, t, s c)}\right) \cdot(1-L)\right]^{\frac{1+\eta}{\eta}}\right\}
$$

In (3), $\mathrm{TD}_{\text {mun(i,t) }}$ is the municipal target demand at the $\mathrm{i}$-th demand center at month $\mathrm{t}$ of the $\mathrm{FH}$; $\operatorname{Def}_{\text {mun }(i, t)}$ is municipal water deficit defined as:

$$
D e f_{\operatorname{mun}(i, t, s c)}=T D_{\operatorname{mun}(i, t, s c)}-\text { Supply } y_{\operatorname{mun}(i, t, s c)}
$$

Moving now to water supply for irrigation, the demand curve for irrigation water is obtained at an irrigation district scale, where typically several different crop types are grown. Common crops grown in the districts considered in the application illustrated in the companion paper include olive trees, vines, citrus, orchard as well as several types of winter and spring vegetables. The demand analysis performed in this study refers to the status quo, both in terms of irrigated crop types and extension and in terms of irrigation efficiency attainable by the irrigation technologies currently in place and the irrigation strategies. It assumes water requirements in a climatically average year.

Besides crop types and irrigated areas, key elements to acquire for each crop to estimate the demand curve include water requirement WRc $\left(\mathrm{m}^{3} / \mathrm{ha} /\right.$ year), crop unit production (ton/ha/year), prices (in $€ /$ ton) at farm gate and the so called specific expenditures SE (€/ha/year). SE are expenses that exhaust their effect during the agricultural year and hence include fertilizers, pesticides, energy, seeds and irrigation water itself, but at the price paid to reclamation consortia, which commonly does not reflect the true value of water. The Italian National Institute of Statistics (ISTAT) now provides the value of the so-called standard output (SO) (€/ha), 
Arena et al., A multi-scenario Decision Support System for real-time operation of over-year multi-reservoir systems 1. Model description

the average monetary value of the agricultural output at farm-gate price, evaluated according to the Eurostat Methodology. It is an average value because it is assessed over a five-year period from farm data pertaining to a whole administrative region. SO is hence computed from crop unit production and crop price. In the spirit of the residual imputation method (Young, 1996), Unit Water Productivity UWPc for the c-th crop type (here in $\left.€ / \mathrm{ha} / \mathrm{m}^{3}\right)$ may then be assessed as UWPc $=(\mathrm{SOc}-\mathrm{SEc}) / \mathrm{WRc}$. UWPc expresses the net value of crop production per $\mathrm{m}^{3}$ of water applied. Water Requirements were obtained from site-specific literature based on the knowledge of water supplied and irrigated areas. As such, they are gross water volumes and do not need to account for losses, as necessary for the municipal sector. Specific Expenditures were obtained by the National Institute of Land Economics (INEA), providing them as a percentage of SO for the area of our application. Multiplying UWPc by the irrigated area Ac in the district, one obtains the water productivity of water $\left(€ / \mathrm{m}^{3}\right)$ for that crop in that district. In districts with multiple crops, the aggregation of the different demands may be performed considering that the available water resources are allocated to crops starting from those with the highest unit value downwards according to non-relaxable constraints of unit water requirements and irrigated areas.

As a result, relationships of the type depicted in Figure 1 may be obtained: each of the boxes represents a different crop-type and circles represent marginal water productivity MWP that decreases when water availability increases. It should be observed that, while in scarcity conditions it may be decided not to grow certain crop types, tree crops such as olive trees, vines, orchard and citrus will necessarily be kept, but their production will be less than in normal years. Studies (e.g. Goldhamer, 2007 for vines and different trees) have shown that for most tree crops there exists a linear relationship between irrigation water and crop yield. This relationship may derived by knowing the fraction $a$ of the production of the fully-irrigated crop when water supply is zero. Considering that when supply $=0$ crop production of the c-th crop is a fraction $\operatorname{Pr}_{c}=a \cdot \operatorname{Pr}_{\max , c}$ and when supply $=W R c$ then $P r_{c}=P r_{\max , c}$, it can be shown that marginal water productivity $\mathrm{MWP}_{\mathrm{c}}$, is given by $(1-a) S O c / W R_{c}$.

Using values of " $a$ " from the available literature on olive trees, citrus and vines, the corresponding marginal values were found and were used in the demand curve for irrigation water.

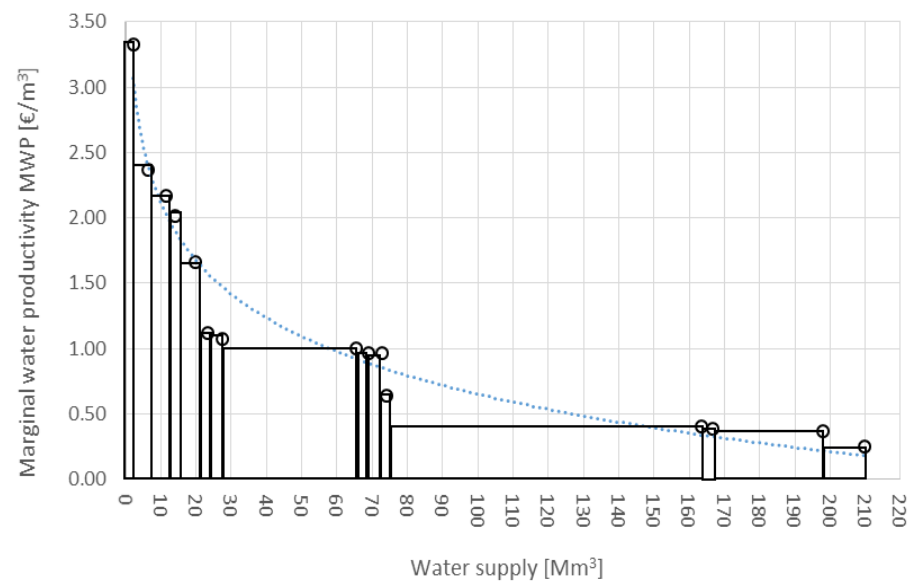

Figure 1. Crops in an irrigation district ordered by water productivity (boxes length indicate crop water requirement at district level) and the interpolated, continuous logarithmic MWP - Water Supply relationship (dotted)

This discrete relationship between marginal water productivity and water supply can be interpolated by a continuous relationship. Using a logarithmic specification of the type for the i-th irrigation centre:

$$
M W P_{(i, s c)}=c_{1(i)}-c_{2(i)} \cdot \ln \left(\text { Supply }_{(i, y r, s c)}\right) c_{1(i)}, c_{2(i)}>0
$$

one obtains, by integration and further passages, the following expression of scarcity costs for the i-th irrigation demand centre:

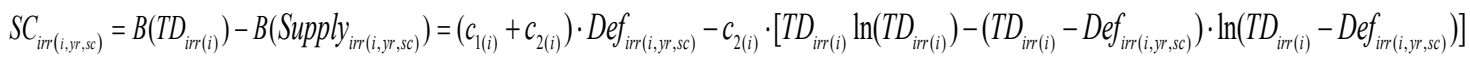

Where $B\left(T D_{\text {irr }(i)}\right)$ is the benefit associated to a water supply that equals target demand $T D_{\text {irr }(i)}$ of the $\mathrm{i}$-th irrigation centre and $\operatorname{Def}_{i r r(i, y r, s c)}$ is the annual deficit of the $\mathrm{i}$-th irrigation centre at year yr in scenario sc. As the 
Arena et al., A multi-scenario Decision Support System for real-time operation of over-year multi-reservoir systems 1. Model description

horizon of decisions is one month, Def $f_{\text {irr }}$ must be defined at both monthly and yearly scale. The related constraints are:

$$
\begin{aligned}
& D e f_{i r r(i, t, s c)}=T D_{\operatorname{irr}(i, t)}-\text { Supply } y_{i r(i, t, s c)} \\
& D e f_{i r r(i, t, s c)}=D e f_{i r r(i, y r, s c)} \cdot \frac{T D_{i r r(i, t)}}{T D_{i r r(i, y r)}}
\end{aligned}
$$

\subsection{Further model constraints}

The proposed model reproduces the topology of the system through an arc-node concept. Hence, besides the above non-anticipatory and definitional constraints, minimization of (1) is also subject to the following constraints:

$$
\begin{aligned}
& S_{(i, t, s c)}=S_{(i, t-1, s c)}+\sum_{j} A d_{(i, j)} \cdot q_{(i, j, t, s c)}+I_{f o r e c a s t(i, t, s c)}+V_{\text {add }(i, t, s c)}-\operatorname{Supply}_{(i, t, s c)}-\operatorname{Spill}_{(i, t, s c)}-\operatorname{Evap}_{(i, t, s c)}-M E F_{(i)} \\
& S_{(i, t, s c)} \leq K_{(i)} \\
& q_{(i, j, t, s c)} \leq q_{\max (i, j)} \\
& q_{(i, j, t, s c)}=q_{(j, i, t, s c)} \\
& \operatorname{Evapo}_{(i, t)}=2.25 \cdot T_{(i, t)}^{1.15} \\
& \operatorname{Area}_{(i, t, s c)}=a_{(i)} \cdot\left[\frac{S_{(i, t-1, s c)}+S_{(i, t, s c)}}{2}\right]^{b_{(i)}} \\
& \operatorname{Evap}_{(i, t, s c)}=\frac{\operatorname{Evapo}_{(i, t)} \cdot \operatorname{Area}_{(i, t, s c)}}{1000} \\
& \operatorname{Supply}_{(i, t, s c)} \leq T D_{(i, t)} \\
& \operatorname{Spill}_{(i, t, s c)} \cdot\left[S_{(i, t, s c)}-K_{(i)}\right]=0 \\
& S_{(i, F H, s c)} \geq S_{f i n(i, F H)} \\
& C_{a d d(i, t, s c)}=U C_{(i)} \cdot V_{a d d(i, t, s c)} \\
& V_{\text {add }(i, t, s c)} \leq V_{\max (i, t)} \\
& S_{(i, t, s c)} \geq 0, q_{(i, j, t, s c)} \geq 0, \text { Supply }_{(i, t, s c)} \geq 0, \text { Spill }_{(i, t, s c)} \geq 0, \operatorname{Evap}_{(i, t, s c)} \geq 0
\end{aligned}
$$

Eqn. (9) is the mass balance at the i-th node of the network between time step t- 1 and time step t of the FH. All terms are dimensionally $\mathrm{Mm}^{3} /$ month. $S_{(i, t, s c)}$ is the storage, if any, at node i. $A d_{(i, j)}$ is the adjacency matrix, an antisymmetric square matrix in which $a_{i j}=1$ if water flows from $i$ to node $j, a_{i j}=-1$ if water flows from node $j$ to node $\mathrm{i}$ and $\mathrm{a}_{\mathrm{ij}}=0$ otherwise. $q_{(i, j, t, s c)}$ is the water flow at time step $\mathrm{t}$ in the arc connecting node $\mathrm{i}$ to node $\mathrm{j}$. $\mathrm{I}_{\text {forecast }(i, t, s c)}$ is the forecasted inflow in the node in scenario sc; it is zero for all nodes that are not reservoirs. $V_{a d d(i, t, s)}$ is the additional water volume entering the node from an additional, costly water resource. Supply $y_{(i, t, s c)}$ is water supplied to the node. It is zero for all the nodes but for demand centers. Spill (i,t,sc) $_{\text {is }}$ water lost from spillways when reservoir storage equals active storage capacity $K$. It can be non-zero only for reservoir-type nodes. $\operatorname{Evap}_{(i, t, s c)}$ are evaporation losses from reservoirs' liquid surfaces. They can be non-zero only for reservoir-type nodes. $M E F_{(i, t)}$ is a constant release for minimum ecological flow. Equations (10) and (11) define maximum storage capacities for nodes and arcs (water supply mains). Eqn. (12) states that flows in the arc 
Arena et al., A multi-scenario Decision Support System for real-time operation of over-year multi-reservoir systems 1. Model description

must be the same, regardless of the starting node. Eqns. (13) to (15) define evaporation losses from reservoirs. $\operatorname{Evapo}_{(i, t)}$ is unit evaporation (in mm) from reservoir's surface, $T_{(i, t)}$ is the average temperature (in ${ }^{\circ} \mathrm{C}$ ) at month $\mathrm{t}$ of the FH. Area $_{(i, t, s c)}$ is the area of the liquid surface (in $\mathrm{Km}^{2}$ ) corresponding to average stored volume in reservoir at time step t. Evap $(i, t)$ is evaporation (in $\mathrm{Mm}^{3} / \mathrm{month}$ ) from reservoirs' liquid surface. Eqn. (16) constraints water supply not to exceed target demand. Eqn. (17) constraints Spill to be 0 if $S_{(i, t, s c)}<$ K. Eqn. (18) is needed to limit the risk to over-allocate resources in the short term: it constraints storage at the final step of the FH not to be under a certain level $S_{\text {fin }}$, specific for each month-type. By avoiding the reservoir to empty at the end of the multi-year planning period, it has proved useful to improve model performances. In eqn. (19) $U C_{(i)}$ is the unit cost of the additional water resources available at site i, $C_{a d d(i, t, s)}$ is the pertaining cost. Volumes from the additional i-th supply source are constrained not to exceed a certain quantity $V_{\max (i, t)}$ that may depend from the month-type. Finally, Eqns. (21) are non-negativity constraints. Model (1) to (21) is non-linear in the objective function (1) and in some of the constraints, namely (14) and (17). To take advantage of the bigger robustness and higher solution speed of linear models the model was linearized. Some highlights of model linearization are reported below. The model is written using the GAMS (General Algebraic Modeling System) platform and is solved using the CPLEX solver embedded in GAMS.

\subsection{Linearization}

\subsubsection{Cost Functions}

Linearization of cost functions in (1) is performed considering the piecewise linearization scheme of Figure 2.

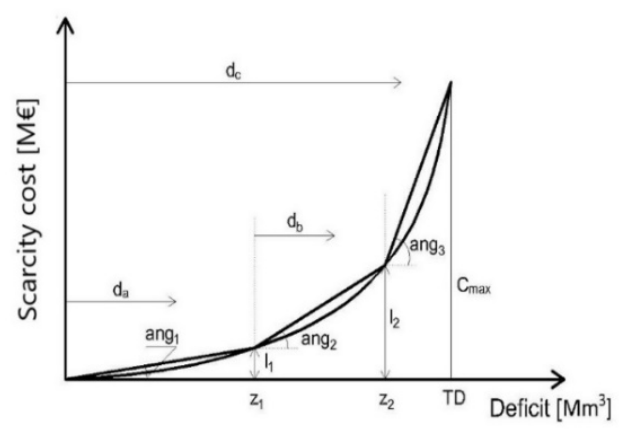

Figure 2. Piecewise linearization scheme for cost functions in the Objective Function

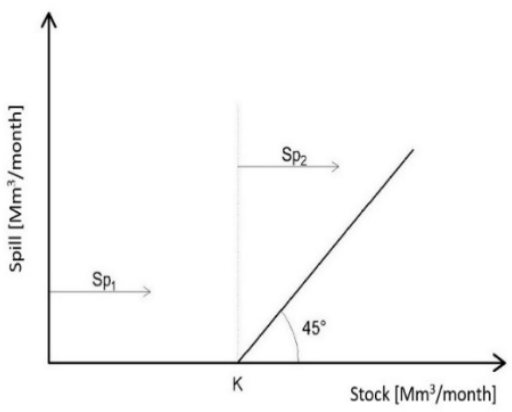

Figure 3. Linearization scheme for the spill function

where $d_{a}, d_{b}$ and $d_{c}$ are real-valued, non-negative variables and bin is a binary decision variable, one for each demand centre and for each time step within the Forecasting Horizon. For brevity sake, the pertaining equations cannot be reported. A similar scheme can be found in Loucks et al. 2005, chapter 4. We deem instead more useful to show actual scarcity costs-deficit relationships developed for the application described in the companion paper (Figures 4 and 5).

\subsubsection{Spills}

Linearization of spill function (17) is instead a somewhat new approach and is reported for this reason. It is performed considering the scheme of Figure 3. First, a "stock" variable is first introduced:

$$
\begin{aligned}
& \operatorname{Stock}_{(i, t)}=S_{(i, t-1)}+\sum_{j} A d_{(i, j)} \cdot q_{(i, j, t)}-\operatorname{Evap}_{(i, t)}-D M V_{(i)} \\
& S p_{1(i, t)} \leq K_{(i)} \cdot Z_{(i, t)} \\
& S p_{2(i, t)} \leq 1000 \cdot\left(1-Z_{(i, t)}\right)
\end{aligned}
$$

In (24) 1000 is a high threshold, necessary for the subsequent (25)

$$
\begin{aligned}
& \operatorname{Stock}_{(i, t)}=S p_{1(i, t)}+K_{(i)} \cdot\left(1-Z_{(i, t)}\right)+S p_{2(i, t)} \\
& \operatorname{Spill}_{(i, t)}=S p_{2(i, t)}
\end{aligned}
$$


Arena et al., A multi-scenario Decision Support System for real-time operation of over-year multi-reservoir systems 1 . Model description

This formulation implies that when $Z_{(i, t)}=1$ (no spill), $S p_{1(i, t)} \leq K_{(i)}$ (Eqn. (23)) and $S p_{2(i, t)} \leq 0$ (Eqn. (24)), hence $S p i l l_{(i, t)}=S p_{2(i, t)}=0$. When $Z_{(i, t)}=0$ (spill is occurring), $S p_{1(i, t)}=0$ and $S p_{2(i, t)} \leq 1000$. From (25) follows that Stock $_{(i, t)}=K_{(i)}+\operatorname{Spill}_{(i, t)}$.

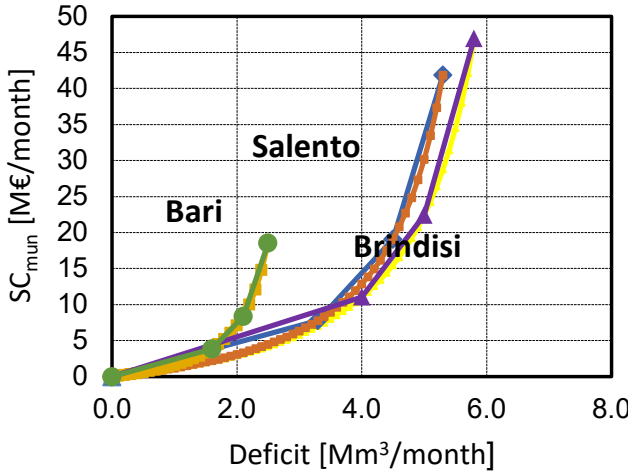

Figure 4. Scarcity costs and piecewise linearization for the municipal demand centres in the application

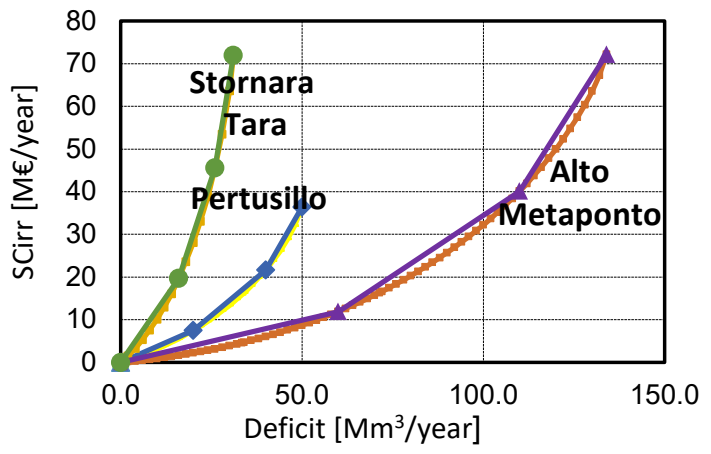

Figure 5. Scarcity costs and piecewise linearization for the irrigation districts in the application

\section{CONCLUSIONS}

The paper has introduced a DSS for the real time operation of multi-reservoir systems where seasonal inflow forecasts are provided by scenarios that are aggregated according to the scenario aggregation principle. The paper has focused on the model description, estimation of scarcity costs and linearization techniques. Its companion paper will discuss estimation issues for some of the relevant parameters of this model (namely, inflows), introduces a case study to illustrate the DSS and benchmark its performances compared to other forecast techniques.

\section{REFERENCES}

Arena, C., Cannarozzo, M., Mazzola, M.R. (2014). Screening Investments to Reduce the Risk of Hydrologic Failures in the Headwork System Supplying Apulia (Italy) - Role of Economic Evaluation and Operation Hydrology. Water Resources Management 28(5), 1251-1275.

Arena, C., Cannarozzo, Oliva, F., M., Mazzola, M.R. (2019). A multi-scenario Decision Support System for real-time operation of over-year multi-reservoir systems 2. DSS simulation. Proceedings MODSIM 2019 Conference, 1-6 December 2019, Canberra, Australia.

Dariane, A.B., Farhani, M., Azimi, Sh. (2018) Long Term Streamflow Forecasting Using a Hybrid Entropy Model. Water Resources Management 32, 1439-1451.

Goldhamer, D.A. (2007). Regulated Deficit Irrigation in Trees and Vines. Agricultural Water Management: Proceedings of a Workshop in Tunisia. https://www.nap.edu/read/11880/chapter/10 last accessed 16.08.19.

Griffin, R. (2006). Water Resources Economics: the analysis of scarcity, policies and projects. The MIT Press, Cambridge Massachusetts, USA.

Loucks, D.P., Van Beek, E., Stedinger, J.R, Dijkman J.P.M., Villars, M.T. (2005). Water Resources Systems Planning and Management - An Introduction to Methods, Models and Applications. Unesco Publishing, Paris, France.

Smith, D.M., Scaife, A.A., Eade, R., and Knight, J. R. (2016). Seasonal to decadal prediction of the winter North Atlantic Oscillation: emerging capability and future prospects. Quarterly Journal of the Royal Meteorological Society 142, 611-617.

Wets R. J.-B. (1989). The Aggregation Principle in Scenario Analysis and Stochastic Optimization Algorithms and Modelling in Mathematical Programming, 91-113. Ed. S. Wallace, Springer-Verlag, Berlin.

Xu, B., Zhong, P.-A., Zambon, R. C., Zhao, Y., Yeh W. W.-G. (2015). Scenario tree reduction in stochastic programming with recourse for hydropower operations, Water Resources Research, 51, 6359-6380, doi:10.1002/2014WR016828.

Young, R.A. (1996). Measuring economic benefits for water investments and policies. World Bank Technical Paper No. 338, World Bank, Washington D.C., USA.

Yuan, X., Wood, E.F., Ma, Z. (2015). A review on climate-model-based seasonal hydrologic forecasting: physical understanding and system development. WIREs Water 2015 2,523-536. 